Discussion

\title{
Comments on "The Marshallian macroeconomic model: A progress report” by Arnold Zellner and Guillermo Israilevich
}

\author{
Antoni Espasa* \\ Statistics Department, Carlos III University, Getafe, Madrid 28903, Spain
}

The paper has two parts. The first one contains a description of the development of the SEMTSA approach since the early 1970s, its applications to macroeconomic variables, the rationalisation of applied models using economic theory, and the extension to disaggregated models within the framework of Marshallian competitive models for production sectors. The second part is devoted to the theoretical derivation of different formulations of the Marshallian macroeconomic model with increasing complexity, starting with a one-sector model with supply, demand, and entry/exit equations, and continuing with models in the single-sector case but extended to demand and supply factor equations to end up with $n$-sector models, which are closed by the inclusion of government and money equations. In this part of the paper, the initial structural models are in differential equation form, leading to reduced form equations useful for forecasting purposes and discrete approximation proposals. In this process, the convergence properties of the main endogenous variables of the differential and difference equation models are illustrated for different values of the parameters and for given initial conditions. It is shown that the difference equation model can approach equilibrium values with oscillatory behaviour and that a wide range of solutions is possible. All this reveals the potential of this theoretical framework for structural analysis, forecasting, and policy simulation in applied macroeconomics.

Professor Zellner is one of the most outstanding econometricians since the late 1950 s, when he published his initial contributions on the consumption function. He has been a highly productive scholar in many fields, such as statistics, econometric theory, methodology, applied econometrics, etc. His research, no matter how theoretical and technical, is always conducted with the aim of producing results which could be used to obtain a better understanding of the real world, and this is why Professor Zellner's research has had such a broad and deep impact on modern econometrics. Today's paper is a very good example of this and is, in fact, highly significant for macroeconometrics. It explains the orientation linking the research in a long sequence of previous papers by the author with a number of important collaborators since the first famous Zellner and Palm paper was published in 1974. These results show that the strategy of developing appropriate and rigorous statisticaleconometric tools for building econometric models using a basic economic theory, which are then fitted to data to test how well they perform and to learn how they can be improved, has been very successful. This 
success is derived from the interaction between empirical and theoretical analysis. Econometric models are initially designed from an economic theory basis but with statistical formulations which are supposed to be appropriate for the characteristics of the data. Then, testing models against data, the analyst learns that some aspects of the economic theory fundamentals are not fully appropriate and obtains clues on how to modify or extend the initial theory. At the same time, data reject some aspects of the statistical structure of the model and these tests tell the analyst which alternative formulations may be more promising. The SEMTSA methodology developed by Professor Zellner has been set up along those lines and, as he concludes, is based on "theory with measurement" and is much to be preferred to others with only one of these two elements.

The large amount of previous research analyzed in the paper is linked by the SEMTSA methodology and its connection with the multi-sector Marshallian macroeconomic model. For the purpose of this paper, we have called it the SEMTSA/MMM approach to applied macroeconometrics.

The $n$-sector Marshallian macroeconomic models give results for the different sectors considered, which can be used to explain the corresponding aggregate variable, such as GDP growth and inflation. Thus the SEMTSA/MMM approach incorporates an important point in the study of macroeconomic variables: With how much disaggregation, if any, should these variables be analysed?

The following remarks will be restricted to some considerations concerning the SEMTSA/MMM approach and its applications in some of the papers mentioned by the authors.

The basic principles in the SEMTSA approach are as follows:

a) Formulation of dynamic models related to a multivariate structural econometric model.

b) Sequential approach to the two main aspects of model building.

c) Keeping models sophistically simple.

The importance of the sequential approach in model building is quite clear in this paper. In one respect, the analyst first produces a model that provides proper forecasting, then tests a model formula- tion capable of explaining the real world, and, finally, attempts to define model specifications which could be entertained for policy and control. An attempt to obtain a model valid for all three of these objectives from the very beginning would lead to failure. Different models could be needed for different goals during this process. In fact, as we discuss later, it is very difficult to initially obtain a model that is useful for different forecasting objectives.

When constructing multivariate models, another sequential approach consisting of first building reliable models for each component, followed by testing the model for the vector of variables, is also advisable.

It has been mentioned that a good strategy in model building is to evaluate the forecasting performance of a given model. Many of the papers in which Professor Zellner and associates applied the SEMTSA or SEMTSA/MMM approach are concerned with point and turning-point forecasting using annual data, in particular turning-points in the GDP growth rate. One of the first papers in this respect is García-Ferrer, Highfield, Palm, and Zellner (1987) and success in forecasting turning-points can also be seen in more recent papers such as Zellner and Min (1999) and Zellner and Tobias (2000).

Reliable turning-point forecasts are very important for investors and institutions, but all these economic agents also demand other forecasting results, even for a given variable like the GDP. In particular, interest often centres on a congruent trace forecast for all time periods in the current and following years. The requirement that the trace forecast must be congruent excludes trace forecasts obtained from different models, minimising the forecast error for a given horizon.

It is not easy to discover how policy makers and practitioners actually evaluate the trace forecasts that they demand. Banerjee, Marcellino, and Masten (2003), following Cecchetti, Chu, and Steindel (2000) for quarterly data and for eight-quarter trace forecasts, adopt an evaluation procedure based on the squared root of the average square forecast errors for one to eight quarters ahead. As pointed out in Banerjee et al. (2003), the elements of this RMSE statistic are highly correlated and a reliable statistical test to discriminate between different forecasting performances is not available. This identifies a question that is as yet unsolved in the literature, important for a more precise application of SEMTSA methodology, 
which is how to evaluate trace forecasts from different models.

The above comments suggest that agents demand updated forecasts each time new data are published. This leads to the need to use quarterly models to study GDP and its components and monthly models for some other variables such as inflation, unemployment, import, export, money aggregate, etc. As has been mentioned in many of the papers published on the methodology, SEMTSA/MMM techniques can be applied to monthly and quarterly time series. But in this case, several additional problems appear. On both levels, monthly and quarterly, the models become more complex because they must take into account the seasonality and short-term dynamics present in the data. But the main problem arises with monthly data. In this case, structural models are often not feasible because some of the relevant explanatory variables are not observed with monthly frequency. The analyst then finds that for forecasting macroeconomic variables which are observed monthly, such as those mentioned above, the best option is generally to construct monthly models. This is so because forecasting accuracy is more enhanced by using recent (monthly) information of the endogenous variable, than by incorporating old (quarterly) explanatory variables data (see Espasa \& Albacete, 2004). On the other hand, quarterly structural models are required to provide an explanation-also highly demanded by the agents-of the forecasts. In these cases, additional econometric devices must be employed to make joint use of the results from monthly reduced form models and quarterly structural ones in order to provide up-to-date forecasts with an economic explanation (see Albacete \& Espasa, 2005 for a particular form of dealing with this question).

The incorporation of the MMM to the SEMTSA approach has provided this methodology with a wide and robust theoretical background from which different types of models can be derived: univariate, single, or multiple equation reduced form and single or multiple equation structural models. This makes this methodology highly flexible, reliable, and useful. A particular advantage should emerge from the analysis of the economy through a consideration of $n$ production sectors. Disaggregating is a way to increase information. What matters is that this information should be really pertinent, and this would be the case if the disaggregation has been guided by economic theory, as in the Marshallian macroeconometric model. In fact, different applications reported in the presentation show significant improvements in forecasting GDP growth from sector forecasts. The limits of the disaggregation have also been mentioned by Professor Zellner. In this respect, it could be said that a disaggregation designed according to economic theory should, in principle, produce more adequate (implied) models for the aggregate and more accurate forecasts. This is what can be expected because more pertinent information has been added to the information set. If this is not the case in a particular application, it must be due to the bad quality of the disaggregated data, or to the complexity involved in building appropriate models for such data. Modelling the components is more difficult than modelling the aggregate directly, not only because of the dimensionality involved, which is obvious, but also because when modelling components, specific exogenous variables, which are not always easily available, often turn out to be crucial. This could explain why the models for agriculture and mining sectors in the MMM provide the worst fits. Another reason for the complexity of disaggregated models is the fact that a linear hypothesis is often bad for some particular components. In these cases, without building nonlinear models, the implied results for the aggregate could be worse than those obtained by modelling it directly. In general, if a component is nonlinear, then the aggregate should be too even when its nonlinearities are less apparent. Therefore, a way to improve the results for the aggregate is to build nonlinear models for the components, when really necessary. In fact, in several instances, it is easier to detect and model the nonlinearities of the aggregate by modelling the particular components which are clearly nonlinear, than by attempting a direct nonlinear model.

A final consideration related to modelling sectors variables refers to whether they can be cointegrated. If this is the case, inclusion of the cointegrated restriction would improve the results. Espasa, Senra, and Albacete (2002) and Espasa and Albacete (2004) show that vector models with cointegration restrictions provide better significant forecasts than aggregated models for inflation in the euro area. Also Minguez and Espasa (2003), breaking down the euro area GDP into four sectors and a fifth component 
corresponding to taxes less subsidies on products, found a significant and important long-run restriction relating the output of services to the outputs of three other sectors, taxes (with a negative coefficient) and a positive trend. They provide evidence that the inclusion of the cointegration restriction improves the forecasts of the aggregate. The disaggregate forecasting approach applied in the Bulletin of EU \& US Inflation and Macroeconomic Analysis uses models for the components of an aggregate including the co-integration relationships between those components.

In conclusion, I would like to say that the SEMTSA/MMM approach is a firm framework for macroeconometric analysis and its different applications have had very encouraging results. The SEMTSA/MMM strategy is not a closed technique, and Professor Zellner has identified the different extensions that he is currently considering. For future developments, some of the aspects mentioned above could be of interest.

\section{References}

Albacete, R., \& Espasa, A. (2005). Forecasting inflation in the euro area using monthly time series models and quarterly econo- metric models. Working paper, Statistics Department, Carlos III University, Madrid, Spain.

Banerjee, A., Marcellino, M., \& Masten, I. (2003). Leading indicators for euro area inflation and GDP growth. Centre for Economic Policy Research, Discussion paper 3893 (http://www. cepr.org/pubs/dps/DP3893.asp).

Bulletin of EU \& US Inflation and Macroeconomic Analysis, number 100, Carlos III University, Madrid, Spain (http:// www.uc3m.es/boletin).

Cecchetti, S., Chu, R. S., \& Steindel, C. (2000, April). The unreliability of inflation indicators. Current Issues in Economics and Finance, 6(4), 16.

Espasa, A., \& Albacete, R. (2004). Econometric modelling for short-term inflation forecasting in the EMU. Working paper, Statistics Department, Carlos III University, Madrid, Spain.

Espasa, A., Senra, E., \& Albacete, R. (2002). Forecasting inflation in the European monetary union: A disaggregated approach by countries and by sectors. European Journal of Finance, 8 , 402421.

García-Ferrer, A., Highfield, R. A., Palm, F., \& Zellner, A. (1987, January). Macroeconomic forecasting using pooled international data. Journal of Business and Economic Statistics, 5(1), 5367.

Minguez, R., \& Espasa, A. (2003). A time series disaggregated model to forecast GDP in the euro-zone. 4th Eurostat and DGECFIN Colloquium, October, Luxembourg.

Zellner, A., \& Min, C. (1999). Forecasting turning points in countries' output growth rates: A response to Milton Friedman. Journal of Econometrics, 88, 203206.

Zellner, A., \& Tobias, J. (2000). A note on aggregation, disaggregation and forecasting performance. Journal of Forecasting, 19, 457469 . 